\title{
OLTARIS: An Efficient Web-Based Tool for Analyzing Materials Exposed to Space Radiation
}

\author{
Tony C. Slaba ${ }^{* a}$, Amelia M. McMullen ${ }^{\mathrm{b}}$, Sheila A. Thibeault ${ }^{\mathrm{a}}$, \\ Chris A. Sandridge ${ }^{a}$, Martha S. Clowdsley ${ }^{a}$, Steve R. Blattnig \\ ${ }^{\text {a }}$ NASA Langley Research Center, Hampton, VA 23681 \\ ${ }^{\mathrm{b}}$ Rochester Institute of Technology, Rochester, NY 14623
}

\begin{abstract}
The near-Earth space radiation environment includes energetic galactic cosmic rays (GCR), high intensity proton and electron belts, and the potential for solar particle events (SPE). These sources may penetrate shielding materials and deposit significant energy in sensitive electronic devices on board spacecraft and satellites. Material and design optimization methods may be used to reduce the exposure and extend the operational lifetime of individual components and systems. Since laboratory experiments are expensive and may not cover the range of particles and energies relevant for space applications, such optimization may be done computationally with efficient algorithms that include the various constraints placed on the component, system, or mission. In the present work, the web-based tool OLTARIS (On-Line Tool for the Assessment of Radiation in Space) is presented, and the applicability of the tool for rapidly analyzing exposure levels within either complicated shielding geometries or user-defined material slabs exposed to space radiation is demonstrated. An example approach for material optimization is also presented. Slabs of various advanced multifunctional materials are defined and exposed to several space radiation environments. The materials and thicknesses defining each layer in the slab are then systematically adjusted to arrive at an optimal slab configuration.
\end{abstract}

Keywords: OLTARIS, HZETRN, space radiation, particle transport, radiation exposure

\section{Introduction}

For near-Earth or deep-space exploration, ionizing radiation is a limiting factor for manned and unmanned missions. The acute and long-term effects of human exposure must be considered to ensure astronaut safety, and sensitive electronic devices that control data transmission, optics, and other research-related or operational tasks must be hardened or shielded to ensure operability over the mission duration. For both humans and electronics, the total exposure can be reduced with increased shielding; however, the additional mass must be considered against other mission constraints such as cost.

Electronic devices are often radiation-hardened to lengthen their operational lifetime or prevent single event effects. For example, computer chips may be manufactured or enriched with alternative materials, and error correcting memory or redundant elements may be utilized. These approaches, coupled to shielding strategies, must be tested to verify the robustness of the system to radiation exposure. Experimental testing can be performed if the radiation environment is simple or well known. However, space radiation environments are a complex mixture of particles including electrons, protons, and heavier ions spanning the periodic table and ranging in energy from a few MeV/n to several GeV/n. The near-Earth radiation environment includes energetic galactic cosmic rays (GCR) that are modulated by solar activity and attenuated by the geomagnetic field, as well as high intensity, low energy belts of trapped electrons and protons, and the potential for solar particle events (SPE). For deep space missions away from planetary bodies, there are no trapped particle environments; however, the exposure from GCR and SPE is increased without the protection of the geomagnetic field. Experimental testing for space systems is therefore difficult and expensive due to the broad range of particles and energies that must be considered.

An approach for testing radiation hardened components or shielding strategies for electronic devices is to supplement experimental testing with results from verified and validated efficient computational tools. This allows

\footnotetext{
*Tony.C.Slaba@nasa.gov; phone 1757 864-1420; fax 1757 864-8094
} 
analyses to be performed rapidly and economically over the broad range of particles and energies found in space. The high efficiency is particularly useful in the early design stages where mass distributions and shielding materials change often. In this paper, we discuss the On-Line Tool for the Assessment of Radiation in Space (OLTARIS), available at https://oltaris.nasa.gov [1]. This tool was developed specifically to provide rapid analysis of materials, shielding concepts, and geometry models in space radiation environments. An overview of the OLTARIS architecture and capabilities is provided, along with results from an example use case with spherical shielding and a simple approach for material optimization.

\section{OLTARIS Overview}

OLTARIS is a web-based set of models that allows scientists and engineers to study the effects of space radiation on humans and electronics. In this section, an overview of the OLTARIS architecture, main modules, and basic functionality is provided. The OLTARIS architecture is divided into two main parts, the website, on which users interact through a browser, and the execution environment, where the computations are performed. The website is built primarily with standard open source components, and the execution environment is primarily FORTRAN executables running on a computational cluster. A flowchart of the data and execution flow is shown in Figure 1. The boxes indicate different components or modules of the system. This modular system makes it easy to maintain and upgrade as new algorithms, methods, and capabilities are developed. The green boxes indicate user-supplied data; the blue boxes indicate data that can either be downloaded from the web server or used in the calculations and stored on the execution host. The gold boxes represent the computations which are performed on the execution host. Each of the boxes will be explained in more detail.

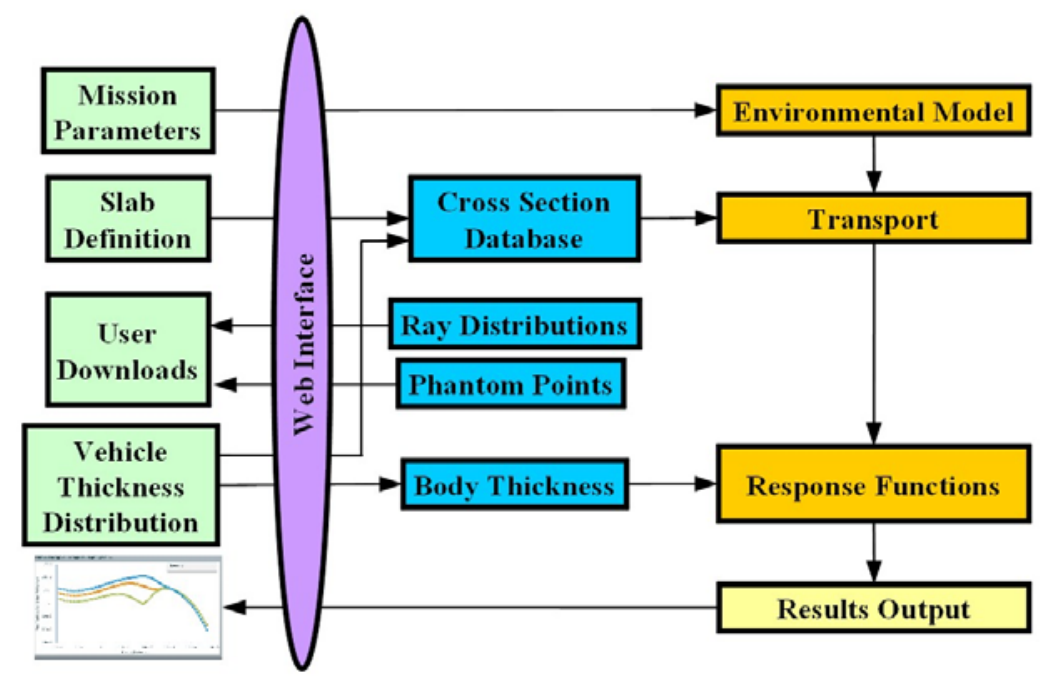

Figure 1. OLTARIS data and execution flow.

The computational engine of OLTARIS is made up of three main modules. The environmental module is used to define an external space radiation environment (boundary condition). The transport module determines how the external environment is modified as it passes through bulk material. The response function module converts the outputs from the transport module to various quantities of interest for biological or electronic related endpoints (dose in silicon, dose equivalent, etc.).

The user can currently select from five different types of space radiation environments: SPE, GCR, Earth orbit, lunar surface, or the Europa design environments. SPE are sporadic events associated with coronal mass ejections made up of protons with energies reaching hundreds of MeV. On OLTARIS, an SPE may be defined in terms of a historical event, linear combination of historical events, or in terms of fitting functions (Weibull fit, exponential in energy, exponential in rigidity, Band function) with user-specified fitting parameters. GCR originate outside our solar system 
and are thought to be accelerated in the shockwaves of supernovae. They are composed of ionized particles spanning the periodic table with energies from a few $\mathrm{MeV} / \mathrm{n}$ up to TeV/n. On OLTARIS, a GCR environment may be defined in terms of historical solar minima or maxima, by mission date, or by a solar modulation parameter. An Earth orbit (circular trajectory) environment may include GCR with an attenuated low energy spectrum due to the geomagnetic field, trapped protons, and albedo neutrons. The trapped proton environment near-Earth is intense but with energies below $100 \mathrm{MeV}$. Albedo neutrons are produced as a result of interactions between the GCR and atmosphere with energies below 100 $\mathrm{MeV}$. On OLTARIS, the Earth orbit environment is defined with a mission time frame, altitude, and inclination. The lunar surface environments are defined in terms of SPE or GCR, and are only applicable for projects with a three dimensional geometry model. In these projects, the radiation from the downward direction (i.e. facing the lunar surface) is neglected. The effect of lunar albedo neutrons is not included at this time. The Europa design environments are selected databases for various Europa mission scenarios including trapped electrons, protons, and heavier ions. The energy spectrum of these particles can extend up to the GeV/n range. A more detailed description of the environment models used in OLTARIS can be found in reference [1].

In order to discuss the transport module, a description of the geometry options within OLTARIS is necessary. There are currently two user-defined geometries that can be analyzed - either a semi-infinite planar slab, or a three dimensional geometry described by a thickness distribution. The semi-infinite planar slab will be referred to simply as a slab throughout the rest of this paper. A slab has a finite thickness in the z direction and infinite thickness in the $x$ and $y$ directions. Slabs may be defined with user-defined materials with any number of layers, in any order, with any finite thickness.

For three dimensional geometry models, a ray-tracing approach is used to provide a distribution of thicknesses and materials surrounding a target point within the geometry. Ray-tracing refers to the process of determining the type and thickness of each material traversed by a ray as it passes from a fixed target point to the geometry boundary. It should be noted that OLTARIS does not provide the capability to ray trace user geometries. The user must perform the ray trace and provide the thickness distribution to the tool. In order to compute the total radiation environment at the point, a large number of rays (usually $\sim 10^{3}$ ) are selected so that the full $4 \pi$ steradians are adequately covered. The distribution of thicknesses along each ray calculated in the ray-tracing procedure is referred to as a thickness distribution. The total exposure (flux or response function) at the point is obtained by using the results from the transport module to obtain an exposure for each ray direction; the total exposure is obtained by integrating the results for all rays. A more detailed discussion of thickness distributions and how they are used within the transport module can be found in the references [1 - 3].

OLTARIS currently allows thickness distributions to have at most three materials in a fixed order. The material types and order may be specified by the user. In complicated geometries, where rays may pass through several types of materials in arbitrary orders, scaling methods $[4,5]$ are often employed by users to convert thicknesses of one material to equivalent thicknesses of another material. No such scaling methods are used implicitly by OLTARIS. In either geometry, users may define or use pre-defined materials (aluminum, polyethylene, silicon, water, tissue, etc.). Userdefined materials may be specified in terms of a chemical formula, elemental mass percentage, or molecular mass percentage. The material definition is used to generate cross section databases that describe probabilities of various atomic and nuclear interactions between projectiles and target atoms and nuclei.

The transport module is composed of two paths depending on the type of geometry the user selects - either slab or thickness distribution. Both paths use transport methods based on HZETRN2010 (High charge (Z) and Energy TRaNsport) [6,7]. For the slab geometry, the chosen boundary condition is transported through user-defined materials and thicknesses to generate flux/fluence at the material interfaces and at the end boundary. For the case of a thickness distribution, the chosen boundary condition is transported through an array of materials and thicknesses to provide a database of flux/fluence values. The database is used in conjunction with a thickness distribution to compute the exposure at a point inside a three dimensional geometry.

The response function module takes the flux/fluence calculated in the transport module and computes selected responses. This includes the differential flux/fluence spectrum, LET (Linear Energy Transfer) spectrum, dose in silicon, dose in tissue, TEPC (Tissue Equivalent Proportional Counter), dose equivalent using the ICRP (International Commission on Radiological Protection) 60 [8] LET dependent quality factor, and effective dose equivalent [9] using the 
human phantoms Computerized Anatomical Man (CAM) [10], Computerized Anatomical Female (CAF) [11, 12], Male Adult voXel (MAX) MAX [13], or Female Adult voXel (FAX) [14]. More detailed discussion of the response functions on OLTARIS can be found in reference [1]. For the case of a slab, the selected response is computed at each layer interface and at the end of the slab. For the case of a three dimensional geometry, the selected response is computed at the target point specified in each thickness distribution. The database of values used to compute the point response within the shielding geometry is also returned.

The website is composed of a series of pages and data that allows various analyses to be created, executed, edited, and examined. The Projects page, shown in Figure 2, is the first page encountered after login. Each project is the complete encapsulation of a calculation; it includes the definition of the external environment, the geometry selection, and response functions. The Projects page is used to create new projects, edit existing projects, submit new jobs to the compute cluster, and access the results of previous jobs. A job is an instantiation of a project that is packaged for processing. When a new project is created, the user is stepped through a series of pages that allows different aspects of the problem to be defined. Help pages are available at any point in the process if more information is needed. Once the project is saved, the user is returned to the Projects page to submit a job to the compute cluster. A project can include multiple jobs so that if particular elements of the project need to be changed (environment, geometry, etc.), the user can do so and create an additional job that can be submitted under that same project.

Once a job is submitted, its status may be queried from the Jobs page. When a job is complete, an email is sent to the user, and the results may be viewed by selecting Display/Download Results for the completed job on the Jobs page. The results page will list some of the results directly in tabular form and allow some data, such as dose versus depth, to be plotted. The Plot page has the option to copy the plotted data to the clipboard so that it can be pasted into various local applications. The user also has the option to download the data to their desktop computer in the form of a text file or can cut-and-paste directly into a spreadsheet.

Another section of the website is the Thickness Distributions page which is selected from one of the main tabs across the top of the page. This page presents a list of the user's current thickness distributions and allows the user to upload new ones. Once a thickness distribution is uploaded to the site, it is available for selection from a Project page. Thickness distributions are uploaded to the site in the form of an XML file. A document describing the format of the file and sample files can be downloaded from the Thickness Distributions page. A phantom Computer Aided Design (CAD) object that represents a human geometry may also be downloaded so that it may be positioned and oriented in the CAD model.

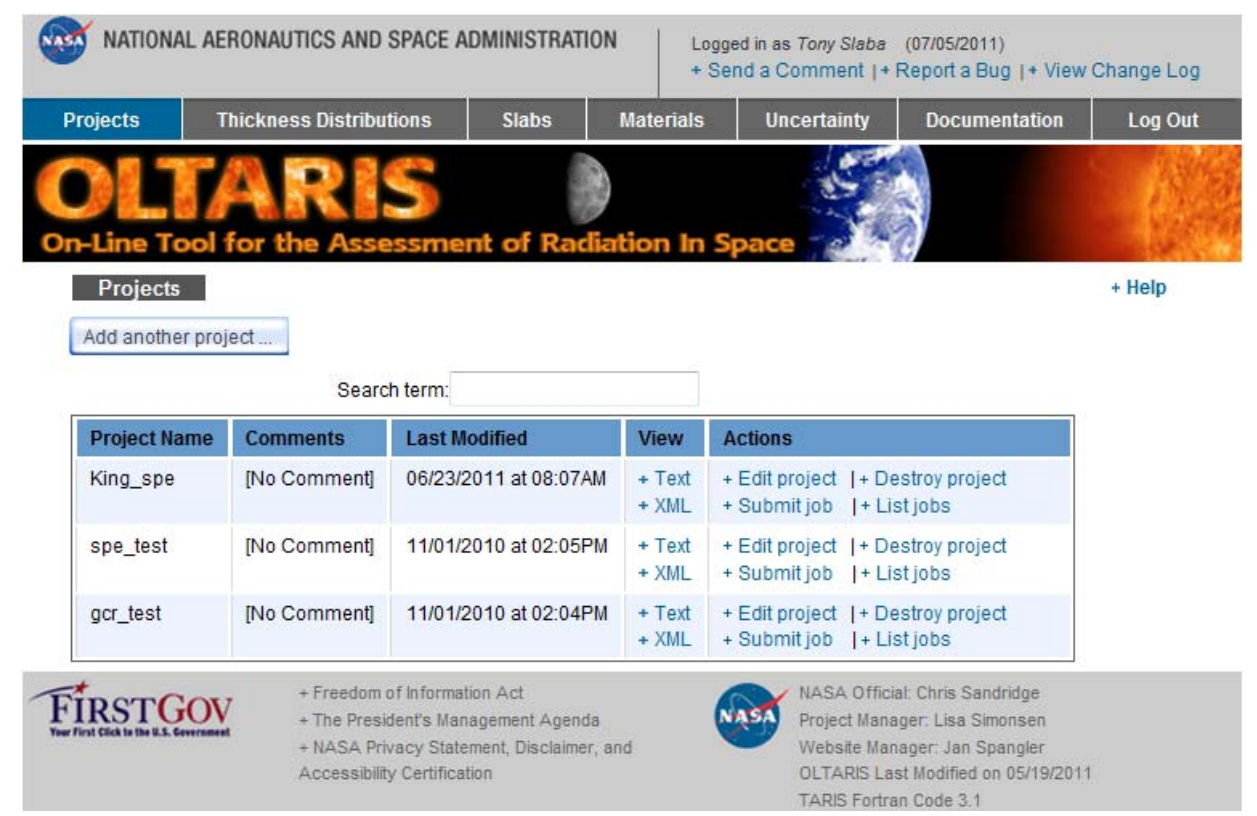

Figure 2. OLTARIS Projects page. 
The Slabs and Materials sections, selected from the tabs at the top of each page, are used to create user-defined slabs of any material. Materials may be defined at the Materials tab by entering the material's elemental mass percentage, its molecular mass percentage, or its chemical formula. Once the material is defined, the definition needs to be submitted to the computational grid so that the cross sections can be computed for later use. Once the cross section database is available, the user can then go to the Slabs tab and define a layup of materials of any thickness, in any order. Once a slab is created, it can be selected for a project from the Projects page. Further discussions regarding the OLTARIS website can be found in the references [1].

\section{Example Use Case: Dose (silicon) versus Depth Material Comparisons}

In early phase mission planning, vehicle, or shield design, it is often useful to compare exposure quantities for various materials as a function of spherical shielding thickness. This allows the radiation shielding properties of materials to be traded against other known properties (mass, thermal, structural, conductive, etc.) in a simple and efficient manner. For electronic systems, a relevant exposure quantity is dose in silicon. In this section, results are presented for ten different materials exposed to SPE and GCR environments with spherical shielding thicknesses reaching $100 \mathrm{~g} / \mathrm{cm}^{2}$. The materials are listed in Table 1 with a brief description. Note that the unit of $\mathrm{g} / \mathrm{cm}^{2}$ is obtained by multiplying the measured length in $\mathrm{cm}$ by the material density in $\mathrm{g} / \mathrm{cm}^{3}$.

Table 1. List of materials exposed to GCR and SPE environments.

\begin{tabular}{ll}
\hline Material & Description \\
\hline ADA3BDA3 & High hydrogen polyimide [15] \\
Aluminum & Pure aluminum \\
Aluminum 6061 & Aluminum alloy with magnesium and silicon \\
Graphite Epoxy 51-49 & Epoxy resin impregnated with graphite particles \\
HD Polyethylene & High density polyethylene \\
Hot pressed BN & Hot pressed boron nitride \\
Tantalum & Pure tantalum \\
Tungsten & Pure tungsten \\
Typical RCC & Reinforced carbon composite \\
Water & Pure water \\
\hline
\end{tabular}

The results are shown in Figure 3 for the 1972 King event [16] (left pane) and the 1977 solar minimum GCR environment [17] (right pane). The King parameterization of the 1972 event is being used due to its historical presence in the literature and use as a design standard environment. The 1977 solar minimum GCR environment is being used also because of its historical presence in the literature and because it represents a severe case for the natural background environment in space. The materials have been ordered in the legend according to the dose level found at $100 \mathrm{~g} / \mathrm{cm}^{2}$. Note that the vertical axis for the SPE results is in log scale, while the vertical axis for the GCR results is linear.

The rapid decline of the SPE dose versus depth curve reflects large energy deposition by the lower energy primary protons as they pass through the slab. Conversely, the slower decline of the GCR dose versus depth curve reflects smaller energy losses by more energetic protons and heavier ions. This indicates that shielding is more effective in reducing exposure levels for SPE than GCR. Figure 3 shows that hydrogen rich materials such as water and polyethylene provide the best radiation shielding at all thicknesses. This is primarily due to the low charge and neutron number in the target (fewer target fragments) and the ability of hydrogen to attenuate low energy neutrons through elastic collisions. Heavier materials such as tungsten and tantalum are less efficient radiation shields due to the large number of target fragments and neutrons that may be produced.

It should be noted that these results are not true for all space radiation environments. For example, lower energy trapped electron environments near-Earth are more efficiently shielded by heavier materials. The data in Figure 3 may also show that there is little difference, from a radiation exposure perspective, between pure aluminum and the aluminum 6061 alloy. This type of information is useful when trading radiation protection properties against other properties 
important for mission objectives (conductive, thermal, structural, etc.). It should be noted that the execution time for the results shown in Figure 3 is on the order of seconds to minutes, thereby allowing many materials to be rapidly analyzed in a variety of environments.
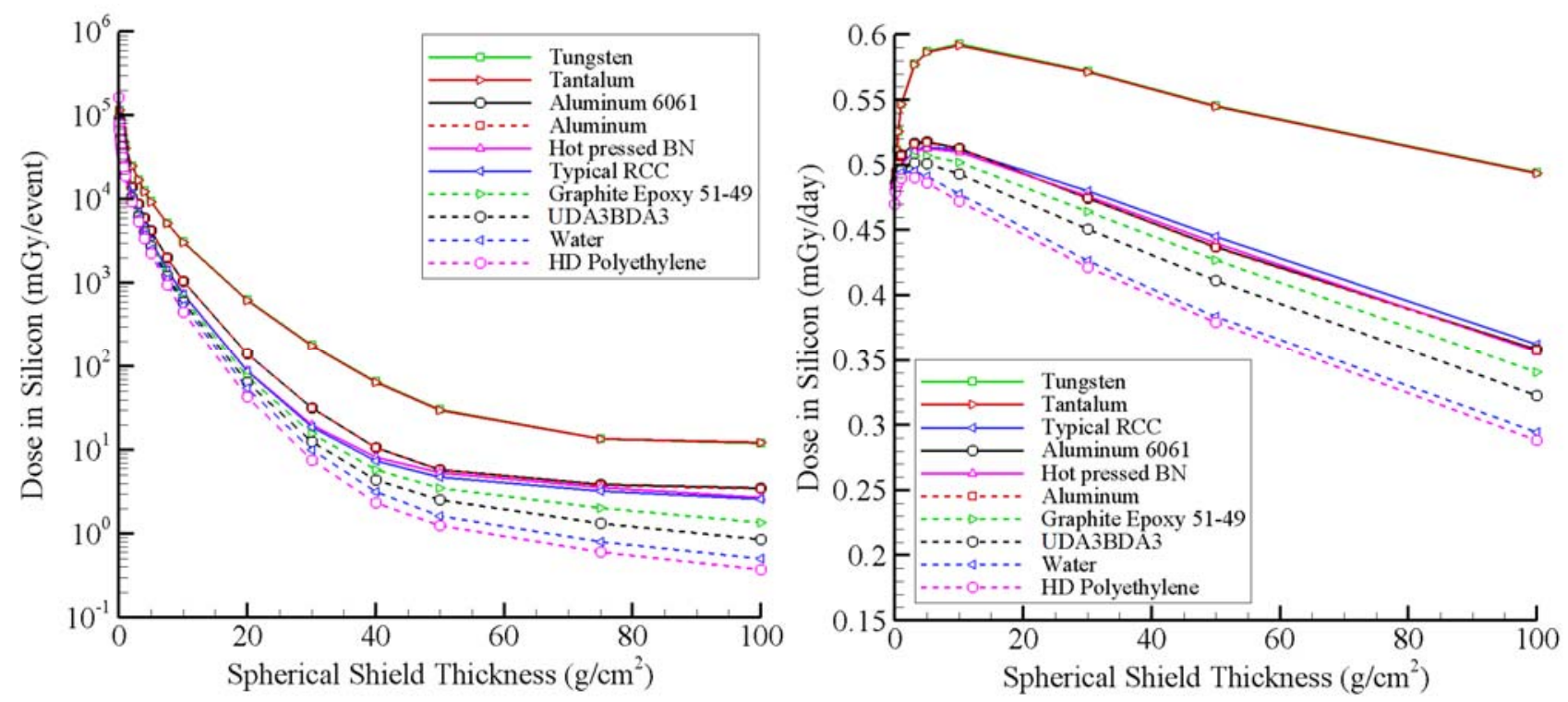

Figure 3. Dose in silicon versus shielding thickness for the 1972 King SPE (left pane) and 1977 solar minimum GCR environment (right pane).

\section{Example Material Optimization}

In this section, an example of material optimization with a simple constraint is provided. This example is not intended to reflect a fully realistic or detailed optimization approach, but rather another demonstration of the ways in which the rapid analysis capabilities of OLTARIS may be utilized.

In space vehicles, habitats, or satellites, external space radiation passes through more than one material type before reaching a sensitive detector or electronic device. In some cases, the ordering of these materials may be flexible and allow an optimal radiation shield design. In order to determine the optimal ordering of the materials, the number of layers, types of materials, and total thickness must be constrained in some way. For this example, aluminum and polyethylene are chosen as the candidate materials and the total slab thickness is fixed at $10 \mathrm{~g} / \mathrm{cm}^{2}$. The optimal shield design is determined by analyzing a variety of slab thicknesses first with aluminum followed by polyethylene (AP) and then with polyethylene followed by aluminum (PA). As in the previous section, the King parameterization of the 1972 SPE and the 1977 solar minimum GCR environments is used. The results are shown in Figure 4.

The horizontal axis represents the fraction of the total slab thickness defined by aluminum. Since the total slab thickness is $10 \mathrm{~g} / \mathrm{cm}^{2}$, an aluminum thickness fraction of 0.25 means that the aluminum layer was $2.5 \mathrm{~g} / \mathrm{cm}^{2}$ and the polyethylene layer was $7.5 \mathrm{~g} / \mathrm{cm}^{2}$. First note that the maximum difference between the AP and PA results is $\sim 5 \%$ for both the SPE (left pane) and GCR (right pane) exposures. The vertical scales in the left and right panes are different, making it appear that the ordering of the materials is more significant for GCR than for SPE. For both cases, the AP configuration provides the lowest exposure, and the minimal exposure is achieved with polyethylene only, as previously suggested in Figure 1. The reduced exposure in the AP configuration is related to the spectrum of particles and energies being produced in the aluminum and subsequently absorbed in the polyethylene. In the AP case, primary particles interact with aluminum and produce low energy target fragments and neutrons that are well attenuated in the hydrogen rich polyethylene. Conversely, in the PA case, primary particles interact with the hydrogen and fewer secondary particles are initially produced. The secondary particles are instead generated in the final aluminum layer and have sufficient energy to exit the slab boundary. 

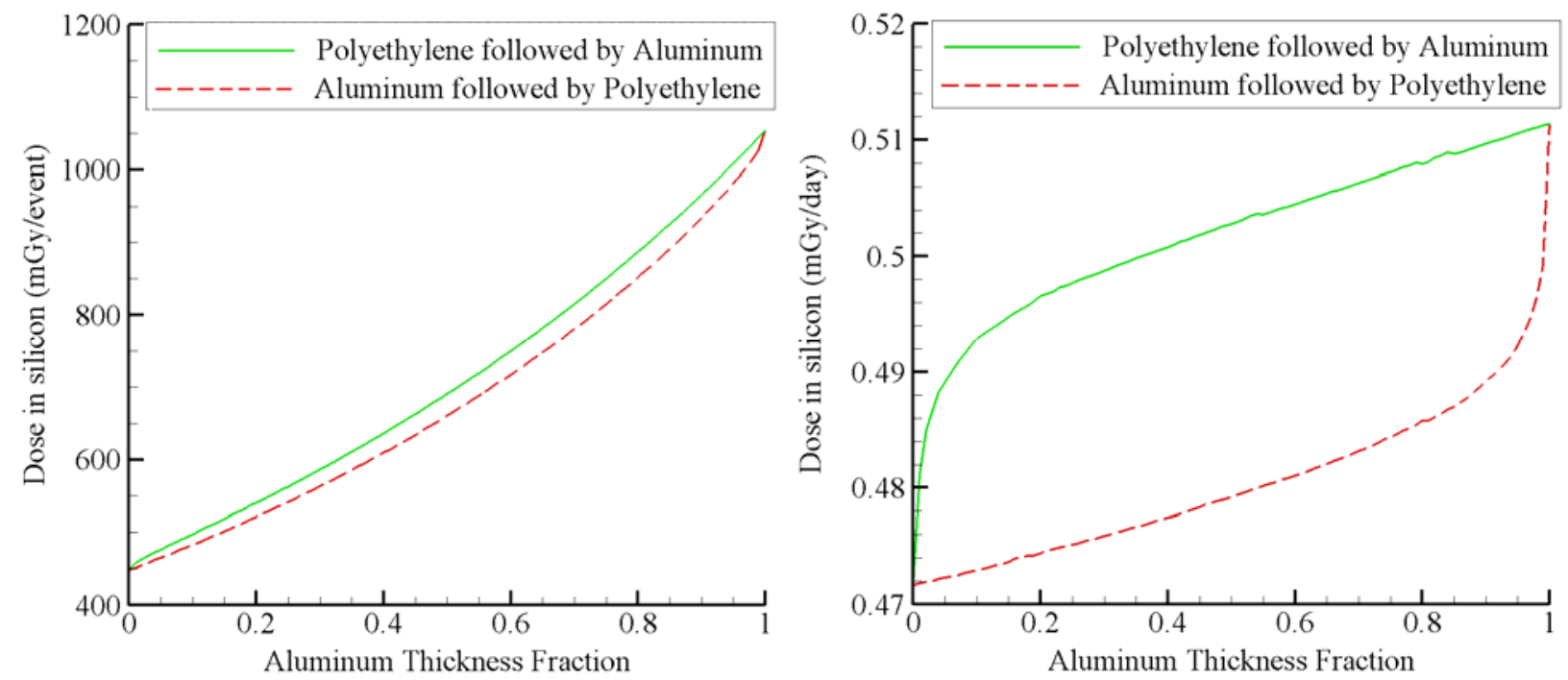

Figure 4. Dose as a function of aluminum thickness fraction at the end of a two layer slab with total thickness of $10 \mathrm{~g} / \mathrm{cm}^{2}$. The slab is composed of aluminum and polyethylene and was exposed to the $1972 \mathrm{King}$ SPE (left pane) and the 1977 solar minimum GCR environment (right pane).

\section{Summary}

The web-based tool OLTARIS available at https://oltaris.nasa.gov is an efficient and accurate tool for researchers and engineers studying the effects of space radiation on humans and electronics. The tool may be used to analyze slab geometries as well as more detailed and complicated three dimensional geometry models through the use of ray-tracing and thickness distributions. Results from two sample use cases were provided here to show the utility of the tool in providing rapid analyses in early phase design or mission planning. However, the tool may be used to provide higher fidelity results as the design and planning phases evolve. Future work will allow users to perform a ray-by-ray analysis of complicated geometries. This approach performs transport along each ray in a thickness distribution with no limit on the number of layers, material types, order, or thickness. Users will also be able to upload trajectory files for near-Earth environments. Analyses will be performed at each point along the trajectory allowing more detailed mission studies.

\section{References}

[1] Singleterry, R.C., Blattnig, S.R., Clowdsley, M.S., Qualls, G.D., Sandridge, C.A., Simonsen, L.C., Slaba, T.C., Walker, S.A., Badavi, F.F., Spangler, J.L., Aumann, A.R., Zapp, E.N., Rutledge, R., Lee, K., Norman, R.B., Norbury, J.W., OLTARIS: On-Line Tool for the Assessment of Radiation in Space. Acta Astronautica, Volume 68, pp. 1086-1097 (2011).

[2] Slaba, T.C., Qualls, G.D., Clowdsley, M.S., Blattnig, S.R., Walker, S.A., Simonsen, L.C., Utilization of CAM, CAF, MAX, and FAX for Space Radiation Analyses using HZETRN. Advances in Space Research, Volume 45 pp. 866-883 (2010).

[3] Slaba, T.C., Blattnig, S.R., Badavi, F.F., Stoffle, N.N., Rutledge, R.D., Lee, K.T., Zapp, E.N., Dachev, Ts. P., Tomov, B.T., Statistical Validation of HZETRN as a Function of Vertical Cutoff Rigidity using ISS Measurements. Advances in Space Research, Volume 47, pp. 600-610 (2011).

[4] Badavi, F.F., Adams, D.O., Wilson, J.W., On the Validity of the Aluminum Equivalent Approximation in Space Radiation Shielding Applications. Advances in Space Research, Volume 46, pp. 719-727 (2010). 
[5] Walker, S.A., Slaba, T.C., Clowdsley, M.S., Blattnig, S.R., Investigating Material Approximations in Spacecraft Radiation Analysis. Acta Astronautica, Volume 69, pp. 6-17 (2011).

[6] Slaba, T.C., Blattnig, S.R., Badavi, F.F., Faster and more Accurate Transport Procedures for HZETRN. Journal of Computational Physics, Volume 229, pp. 9397-9417 (2010).

[7] Slaba, T.C., Blattnig, S.R., Aghara, S.K., Townsend, L.W., Handler, T., Gabriel, T.A., Pinsky, L.S., Reddell, B., Coupled Neutron Transport for HZETRN. Radiation Measurements, Volume 45, pp. 173-182 (2010).

[8] International Commission on Radiological Protection. Recommendations of the International Commission on Radiological Protection. ICRP Publication 60, Pergamon Press, New York (1991).

[9] NCRP, Operational Radiation Safety Program for Astronauts in Low-Earth Orbit: A Basic Framework. NCRP Report 142, Bethesda, MD (2002).

[10] Billings, M.P., Yucker, W.R., The Computerized Anatomical Man (CAM) Model. Summary Final Report, MDCG4655, McDonnell Douglas Company (1973).

[11] Yucker, W.R., Huston, S.L., The Computerized Anatomical Female. Final Report, MDC-6107, McDonnell Douglas Company (1990).

[12] Yucker, W.R., Reck, R.J., Computerized Anatomical Female Body Self-Shielding Distributions. Report, MDC 92H0749, McDonnell Douglas Company (1992).

[13] Kramer, R., Vieira, J.W., Khoury, H.J., Lima, F.R.A., Fuelle, D., All about MAX: A Male Adult Voxel Phantom for Monte Carlo Calculations in Radiation Protection Dosimetry. Physics in Medicine and Biology, Volume 48, pp. 1239-1262 (2003).

[14] Kramer, R., Vieira, J.W., Khoury, H.J., Lima, F.R.A., Loureiro, E.C.M., Lima, V.J.M., Hoff, G., All about FAX: A Female Adult Voxel Phantom for Monte Carlo Calculations in Radiation Protection Dosimetry. Physics in Medicne and Biology, Volume 49, pp. 5203-5216 (2004).

[15] Orwell, R.A., College of William and Mary, private communication (2011).

[16] King, J.H., Solar Proton Fluences for 1977-1983 Space Missions. Journal of Spacecraft and Rockets, Volume 11, pp. 401-408 (1974).

[17] O’Neill, P.M., Badhwar-O’Neill Galactic Cosmic Ray Model Update Based on Advanced Composition Explorer (ACE) Energy Spectra from 1997 to Present. Advances in Space Research, Volume 37, pp. 1727-1733 (2006). 